\title{
DNA replication, for a change
}

Genome rearrangements that lead to human disorders are typically thought to be caused by mistakes that occur during recombination. A new study into the genetic basis of a CNS disorder has revealed that, in fact, some disease-causing changes in genomic structure might arise from errors that happen during DNA replication, suggesting a new mechanism for generating structural variation in the human genome.

Most genomic disorders occur by non-allelic homologous recombination (NAHR), in which illegitimate meiotic exchange is promoted between low-copy repeats (LCRs) or larger repetitive regions, leading to clustered mutation breakpoints, deletions or duplications. Although this model holds well for recurrent rearrangements, the mechanistic basis of non-recurrent genome changes is less well understood, but some appear to occur by non-homologous end joining (NHEJ). To address this issue, the authors carried out a high-resolution analysis of breakpoint sequences in patients suffering from Pelizaeus-Merzbacher disease (PMD), an X-linked dysmyelinating disorder that is associated with variable and non-recurrent structural mutations in the proteolipid protein 1 gene $(\underline{P L P 1})$.

The breakpoints of 17 patients with $\mathrm{PMD}$, and their informative relatives (where appropriate), were analysed using a combination of PCR-based sequence amplification and high-resolution comparative genomic hybridization (CGH) using an oligonucleotide array. Although some of the breakpoint junctions of recurrent rearrangements contained simple duplications - as you would expect as a result of NHEJ - most rearrangements were more complex, involving stretches of duplication interspersed with normal copy-number sequences and deletions.

A purely recombination-based mechanism, such as NAHR or NHEJ, cannot explain such patterns of rearrangement. However, the authors pieced together the location of the breakpoint junctions, and the identity and orientation of the rearranged sequences, and proposed a mutation

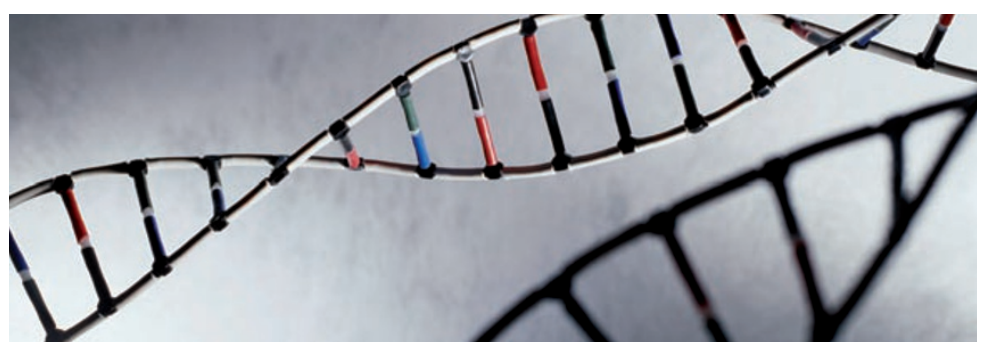

model that invokes aberrant replication. The PLP1 gene is surrounded by LCRs of various sizes - these would make it easy for the fork to stall during replication and then for the lagging strand to associate serially with the DNA-template strand of another active fork, before returning to the original template. This model, which the authors call FoSTeS (fork stalling and template switching), would require only small stretches of homology but could involve sequences that are megabases apart.

The model itself is not entirely new - aspects of it have already been proposed to explain gene amplification in bacteria and other gene rearrangements (such as inversions) in eukaryotes - but this is the first time that such a replication mechanism has been found to drive a disease process. Given the growing recognized importance of small-scale structural variation, it is conceivable that errors during replication contribute not only to other genomic disorders but also to inducing changes, such as gene duplications, that have short- and long-term evolutionary consequences.

ORIGINAL RESEARCH PAPER Lee,, . A. et al. A DNA replication mechanism for generating nonrecurrent rearrangements associated with genomic disorders. Cell 131, 1235-1247 (2007) FURTHER READING Feuk, L. et al. Structural variation in the human genome. Nature Rev. Genet. 7, 85-97 (2006) 